\title{
Differentiated Semantic Analysis in Lexical Affect Sensing
}

\author{
Alexander Osherenko
}

\author{
Elisabeth André
}

\author{
University of Augsburg \\ Germany, Eichleitnerstr. 30, 86159 Augsburg \\ [osherenko, andre]@informatik.uni-augsburg.de \\ http://emotext.informatik.uni-augsburg.de
}

\begin{abstract}
Recently, there has been considerable interest in the recognition of affect from written and spoken language. In this paper, we describe an approach to lexical affect sensing that performs a semantic analysis of texts utilizing comprehensive grammatical information. Hereby, the proposed approach differentiates affect of many classes. In addition, this paper reports on obtained results and discusses them.
\end{abstract}

\section{Introduction}

Lexical affect sensing is an important field of study with a high potential in many application fields, for instance, in robotics or tutoring systems. However, emotional analysis of texts presents a great challenge to computer scientists due to the variety of emotional texts and the vagueness of expressed meanings.

There are two types of approach that implement lexical affect sensing: statistical and semantic. Statistical approaches make use of data mining methods, e.g. Support Vector Machines (SVM) and classify emotion in text, for instance, by using word counts [14]. However, statistical approaches frequently rely on shallow information such as keyword or word category counts whose influence on affective meaning is not always humanly plausible.

In contrast, semantic approaches utilize the meanings of words such as negations, and rely on common sense knowledge such as the fact that an accident is an unpleasant event. For instance, Prendinger and colleagues [13] describe a semantic approach to affect sensing using 160 sentences from a corpus with weblogs annotated with one of nine Izard's emotions and a corresponding intensity value. The approach utilizes a database that comprises of 1,627 emotion words extracted from WordNet-Affect [19], 362 empirical emoticons, 337 most popular acronyms and abbreviations. The entries in the database are manually annotated as 10-element vectors of Izard's emotions [8]. The proposed algorithm uses 112 modifiers of emotional meanings as intensifiers, e.g. very, and negations, e.g. not. For simplicity, texts starting with think, believe, sure, know are dropped and sentences starting with if, when, whenever, after, before are discarded because they express a modal attitude towards the proposition. The proposed approach analyses emotional meaning of a sentence in five stages: 1) the approach looks for emoticons in the studied text and, if found, completes the algorithm assuming that emotional meaning of the analyzed text is conveyed by the emotional meaning of these emoticons; 2) emotional phrases of the text are extracted; 3) the emotional meaning of emotion words is extracted as an Izard's vector from the system database as well as empirical weights of encountered modifiers; 4) the emotional meaning of adjective, noun, verb phrases is calculated; 5) the approach calculates emotional meaning considering emotional phrases and the grammatical structure of the analyzed text. The approach is assessed by 3 annotators: the output agrees with the result of one annotator in $79.4 \%$ of the sentences, and with the result of at least 2 annotators in $70 \%$ of the sentences.

Liu and colleagues [12] describe an approach to affect sensing at the sentence/phrase level based on commonsense facts, e.g. Some people find ghosts to be scary. The proposed approach extracts all sentences from Open Database Common Sense database which contain emotional known seed words, for example, the emotional adjective happy. Then, the approach generates sentence models and calculates the corresponding Ekman vector. Evaluation of the prototype system (an e-mail client called EmpathyBuddy) is done by a usability test with 20 users that assess the system qualities as entertainment, interactivity, intelligence, adoption and confirm a satisfactory system performance.

Wiebe and Riloff [20] describe an approach to automatically classifying sentences as subjective using an unannotated corpus that consists of 298,809 sentences from a world press collection. The approach uses the empirical knowledge that distinguishes strong and weak clues of subjectivity.

The approach by Choi and Cardie [4] considers compositional semantics to analyze affect in sentences: the meaning of a compound expression is a function of its parts and of the syntactic rules by which they are combined. In order to analyze the emotional meaning of phrases they differentiate between the emotional meaning of particular phrases.

Our approach utilizes the approaches above as 
follows: affect sensing considers emotion words extracted from dictionaries of affect, e.g. from WordNet-Furthermore, we utilize empirical knowledge to calculate emotional meaning of texts and consider hereby compositional semantics: our approach calculates the classification result by splitting the whole text in parts; the emotional content of the whole text is derived from the results obtained for the single parts where particular words "intensify" the emotional meaning. However, we extend previous approaches in several respects. Unlike Prendinger and colleagues, we do not just make use of a syntactic parser, but combine syntactic with semantic parsing to derive emotional content. In addition to phenomena discussed by earlier work, such as intensifiers, we handle grammatical rules and consider the role of exclamatory and rhetorical questions in order to capture emotional meaning of text. For example, we are able to distinguish between "Never before I was so happy" and "I was never happy." The emotion word and the negation word are in both cases the same, but the emotional meaning is completely different. The coding of rules for capturing such phenomena is facilitated by the integration of a syntactic and semantic parser. Our rules are motivated by linguistic studies such as the study by Leech and Svartvik [10] and by own corpus studies.

\section{Affective Sources}

This section describes sources of affective information utilized in the proposed approach.

\subsection{Emotion Words from Affect Dictionaries}

In our study, we consider 4,527 affect words: 503 words from WordNet-Affect, 34 Levin verbs [11], 3990 words from GI [17] (1,790 positive and 2,200 negative).

\subsection{Movie Glossary}

Our approach analyzes sentences of movie reviews that usually contain movie titles. Since movie titles do not convey affect, but could be erroneously interpreted as affective an approach to affect sensing should consider movie titles in order to avoid confusion.

Hence, we define concepts obtained from the movie review glossary [7]. For example, the word actor or movie titles are concepts from the movie review domain. In our approach, concepts convey the neutral emotional meaning. For instance, the sentence Actually, one of the biggest problems with Happy Gilmore is that it pretends to have a plot expresses negative affect: its emotional meaning is expressed by the word problems. However, this sentence contains the positive emotion word happy in the movie title Happy Gilmore that could be confused with an affect-bearing word. To avoid these problems, our approach maintains the movie titles from the studied corpora and 15 concepts from the movie domain.

\section{Grammatical Sources}

The sections below show grammatical information sources utilized for the semantic analysis of texts.

\subsection{Means from Linguistic Literature}

The linguists Leech and Svartvik describe 11 grammatical rules to scrutinize the emotional meaning of texts. These rules are considered in the proposed approach as intensifiers of emotional meaning:

1. Interjections, e.g. Oh, what a beautiful present! The interjection $O h$ intensifies the emotional meaning of the emotion word beautiful.

2. Exclamation, e.g. What a wonderful time we've had! The exclamation intensifies the meaning of the emotion word wonderful.

3. Emphatic so and such, e.g. I'm so afraid they'll get lost! The emotional meaning of the sentence is modified by the intensifiers so and such.

4. Repetitions, e.g. This house is 'far, 'far too expensive! In this example, repetitions of the word f $f a r$ are considered as an intensification of the negative meaning of the word expensive.

5. Intensifying adverbs and modifiers, e.g. We are utterly powerless. In this example, the adverb utterly intensifies the negative meaning of the word powerless.

6. Emphasis, e.g. How ever did they escape? In this example, the emphasis word ever is considered as an intensifier of the negative meaning of the emotion word escape.

7. Intensifying a negative sentence, e.g. She couldn't avoid this trouble at all. In this example, the phrase at all in the negated sentence is considered as an intensifier of the negative meaning of the emotion word avoid;

8. A negative noun phrase beginning with not $a$, e.g. We arrived not a moment too soon. In the example, the phrase not $a$ is considered as an intensifier.

9. Fronted negation, e.g. Not a penny of the money did he spend. In the example, a negation, such as not, at the beginning of the sentence is considered as an intensifier.

10. Exclamatory question, e.g. Hasn't she grown!. Since every studied text ending with an exclamation mark can be considered without loss of generality as an exclamatory question, every occurring exclamation mark is considered as an intensifier.

11. Rhetorical questions, e.g. What a difference does it make?. Since every studied text ending with an question mark can be considered without loss of generality as a rhetorical question, every occurring question mark is considered as an intensifier. 
Note that not all examples we mentioned above contain a word that bears an evident emotional meaning. Some of them rather include words that intensify a meaning, such as the fronted negation not a. Here, the emotional meaning is conveyed by other means, e.g. by the context.

\subsection{Means from Own Corpus Studies}

Based on the analysis of emotional texts containing negations and intensifiers, we collected rules for analyzing the emotional content of sentences. For this purpose, we use 74 intensifiers as well as negation words, e.g. not, never, any, almost from [15].

\section{Differentiated linking of emotional parts}

The emotional content of texts can be analyzed at different levels of granularity. In order to reduce significantly complexity, an approach to semantic affect sensing can split a text into parts (subsentences, phrases) and analyze the emotional meaning of the parts independently. Finally, the emotional meanings of the single parts can be combined in order to calculate emotional meaning of the whole text.

To determine the emotional meaning of the complete text, our system considers both combinations of subsentences and phrases the text is composed of where we distinguish between superordinate and subordinate clauses.

Let us have a look at an example of affect sensing given three classes of affect: positive, negative, neutral. We split the text Alexander is very sad, but everybody else is happy in two subsentences: <negative superordinate clause $><$ positive superordinate clause $>$. The meaning <negative superordinate sentence $>$ is calculated on the basis of the negative meaning of the emotion word sad. The meaning <positive superordinate clause $>$ is calculated on the basis of the positive meaning of the emotion word happy. The emotional content of the phrases for the superordinate clause Alexander is very sad is <neutral $><$ neutral $>$ $<$ negative $>$; the emotional content of the phrases for the superordinate clause but everybody else is happy is $<$ neutral $><$ neutral $><$ neutral $><$ positive $>$. The emotional content of the text $<$ negative superordinate clause $><$ positive superordinate clause $>$ is assumed as negative.

Now let us analyze the text Alexander is very sad, but everybody else is happy using five classes of affect: high positive, low positive, high negative, low negative, neutral. The text yields the following emotional content of two subsentences: <high negative superordinate clause $><$ low positive superordinate clause $>$ that can be summed up as the low negative emotional content. The meaning <high negative superordinate clause $>$ is calculated on the basis of the negative meaning of the emotion word sad intensified through the word very. The meaning <low positive superordinate clause $>$ is calculated on the basis of the positive meaning of the emotion word happy. The emotional content of the phrases Alexander is very sad is <neutral $><$ neutral $>$ $<$ high negative $>$ that ascribes the high negative emotional content for the superordinate clause; the emotional content of the phrases but everybody else is happy is <neutral $><$ neutral $><$ neutral $><$ low positive $>$ that ascribes the low positive emotional content for the superordinate clause. Emotional content of the text $<$ high negative superordinate clause $><$ low positive superordinate clause $>$ is assumed to be low negative.

\section{Corpora}

In our study, we used two corpora: FWF and BMRC$\mathrm{S}$. These corpora are characterized by grammatically correct sentences. Hereby, we disregard issues of context and anaphora resolution.

We choose in our experiments the Fifty Word Fiction corpus (FWF) containing 759 English sentences that are manually annotated in terms of their sentiment and affect as positive, neutral, or negative [16]. For instance, the corpus contains the sentence We all laughed and ordered beers which is annotated as positive. The corpus was collected online and available to the general public for one month, during which some 3,301 annotations were made by 49 annotators. 82 sentences are annotated as positive, 171 sentences as negative, and 506 sentences as unclassifiable. The sentences were annotated using the majority vote of the annotators yielding the inter-coder agreement value of $65 \%$. This value is less than $80 \%$ - a desirable agreement following [4] - however, FWF is used in this study for the reason of its free availability.

In order to examine our approach, we compiled a corpus with sentences from 13 movie reviews Berardinelli Movie Review Corpus - Sentences (BMRC-S) [1]. BMRC-S contains both plot sentences with movie details as well as subjective sentences that convey opinion. For instance, BMRC-S contains the sentence A Clockwork Orange is told in three acts that describes a film (Clockwork Orange is a movie title); in addition, BMRC-S contains the sentence In this role, Léaud is fantastic! that expresses an opinion about the performance of the actor Léaud.

BMRC-S was compiled by splitting up the movie reviews in sentences; each sentence was annotated manually using a 5-class annotation scheme: the high negative sentences correspond to the sentences with low valence and high arousal, the low negative sentences correspond to the sentences with low valence and low arousal, the neutral sentences correspond to the sentences with the evaluation and arousal values around zero, the high positive sentences correspond to the sentences with low valence and low arousal, the low 
positive sentences correspond to the sentences with low valence and low arousal. The resulting 5-classes BMRC-S contains 1,010 emotional sentences: 173 sentences were annotated as high negative, 432 sentences as low negative, 169 sentences as neutral, 65 sentences as high positive, 171 sentences as low positive. On the basis of the 5-classes annotation, a 3classes annotation (negative, neutral, positive) is calculated that transforms more detailed 5-classes annotation in less detailed 3-classes annotation by changing low/high positive annotation in positive annotation; low/high negative annotation is changed in negative annotation; the neutral annotation remains neutral. Hence, 605 sentences in the 3-classes BMRC-S are annotated as negative, 169 sentences as neutral, 236 sentences as positive.

\section{System}

We developed a computer system that implements semantic affect sensing by utilizing the information above. The system uses the SPIN parser for performing order-independent word matching [6] and the Stanford parser for POS-tagging and detection of parts [9].

The system uses SPIN rules that match particular words in texts and considers hereby grammatical information. It maintains 4,527 rules for matching emotion words and 20 rules to process film titles. Furthermore, it uses 154 grammatical rules: 18 rules to capture information from the literature (see Section 3); 20 rules from our own investigations; 19 rules for linking phrases (phrase combinations of different emotional content); 97 rules for homogenous /heterogeneous linking superordinate/subordinate clauses of different emotional content. For instance, we use a 5-classes rule <high negative superordinate clause $><$ low positive superordinate clause $>$ implies $<$ low negative affect $>$ for heterogeneous linking of a superordinate and the subordinate sentence that ascribes the sentence a low negative emotional content.

The system distinguishes text fragments of three granularities using the Stanford parser: (1) the whole text - there is no splitting; (2) a division of the text into subsentences; (3) a division of the text into phrases. Additionally, the majority vote is calculated on the basis of three granularity votes.

To recognize affect in a text fragment of a particular granularity, the system performs word-spotting and distinguishes hereby three strategies: (1) the first phrase word-spotting strategy considers the emotional meaning of a first emotional phrase occurring in a text as the emotional meaning of the whole text; (2) the last phrase word-spotting strategy considers the meaning of a last emotional phrase in the text fragment as the resulting emotional meaning; (3) the average vote word-spotting strategy calculates the meaning of a whole text as an average meaning of all emotional phrases in the analyzed text. For instance, the phrase I am happy and sad containing the emotion word happy and the emotion word sad is analyzed according to the first phrase wordspotting strategy as positive represented by the emotion word happy; an emotion word sad defines according to the last phrase word-spotting strategy the negative meaning of the text; according to the average vote word-spotting strategy the text is neutral (there is no emotional majority, happy vs. sad).

Given a text granularity and a word-spotting strategy, the algorithm for semantic affect sensing works as follows:

1. Chosen text granularity is 'whole text': The approach detects emotion words in the analyzed text and classifies its emotional meaning according to the chosen word-spotting strategy.

2. Chosen text granularity is 'subsentences'. The approach extracts superordinate and subordinate subsentences and classifies their emotional meaning by applying the chosen word-spotting strategy; afterwards it constructs a corresponding subsentence combination and classifies the emotional meaning of the analyzed text by applying linking rules.

3. Chosen text granularity is 'phrases'. The approach performs affect sensing of texts in two nested steps: first, emotional meanings of subsentences are calculated using phrase combinations which emotional meanings are calculated under consideration of the utilized word-spotting strategy; second, the emotional meaning of a text is calculated by constructing a subsentence combination on the basis of the calculated phrase combinations.

4. Chosen text granularity is 'majority'. The approach considers the majority of affect votes yielded by the granularities above as result of affect sensing.

\section{Results}

Table 1 shows results of the proposed approach to semantic affect sensing as a 3-classes problem. The Corpus column presents the tested corpus (FWF or BMRC-S); the Bln. column is the class-wise baseline recall value calculated using the statistical approach in [14]; the Gran. column represents the granularity of the text division (either whole text, subsentences, phrases, or the majority vote); the Strategy column shows the utilized word-spotting strategy (first phrase, last phrase, average vote); the $R$ column shows the class-wise recall value; the $P$ column shows the class-wise precision value. 


\begin{tabular}{|c|c|c|c|c|c|}
\hline Corpus & Bln. & Gran. & Strategy & $R$ & $P$ \\
\hline \multirow{12}{*}{$F W F$} & \multirow{12}{*}{37.20} & \multirow[t]{3}{*}{ Majority } & $\begin{array}{l}\text { First } \\
\text { phrase }\end{array}$ & 47.20 & 44.09 \\
\hline & & & $\begin{array}{l}\text { Last } \\
\text { phrase }\end{array}$ & 47.64 & 44.26 \\
\hline & & & $\begin{array}{l}\text { Average } \\
\text { vote }\end{array}$ & 45.92 & 43.14 \\
\hline & & \multirow[t]{3}{*}{$\begin{array}{l}\text { Whole } \\
\text { Text }\end{array}$} & $\begin{array}{l}\text { First } \\
\text { phrase }\end{array}$ & 45.41 & 42.90 \\
\hline & & & $\begin{array}{l}\text { Last } \\
\text { phrase }\end{array}$ & 47.45 & 44.05 \\
\hline & & & $\begin{array}{l}\text { Average } \\
\text { vote }\end{array}$ & 42.79 & 41.15 \\
\hline & & \multirow[t]{3}{*}{ Subsent. } & $\begin{array}{l}\text { First } \\
\text { phrase }\end{array}$ & 47.20 & 44.08 \\
\hline & & & $\begin{array}{l}\text { Last } \\
\text { phrase }\end{array}$ & 47.24 & 44.03 \\
\hline & & & $\begin{array}{l}\text { Average } \\
\text { vote }\end{array}$ & 46.04 & 43.22 \\
\hline & & \multirow[t]{3}{*}{ Phrase } & $\begin{array}{l}\text { First } \\
\text { phrase } \\
\end{array}$ & 44.79 & 42.90 \\
\hline & & & $\begin{array}{l}\text { Last } \\
\text { phrase }\end{array}$ & 45.21 & 43.13 \\
\hline & & & $\begin{array}{l}\text { Average } \\
\text { vote }\end{array}$ & 44.22 & 42.41 \\
\hline \multirow{12}{*}{$B M R C-S$} & \multirow{12}{*}{52.34} & \multirow[t]{3}{*}{ Majority } & $\begin{array}{l}\text { First } \\
\text { phrase }\end{array}$ & 54.18 & 51.39 \\
\hline & & & $\begin{array}{l}\text { Last } \\
\text { phrase }\end{array}$ & 53.33 & 50.45 \\
\hline & & & $\begin{array}{l}\text { Average } \\
\text { vote }\end{array}$ & 52.11 & 48.72 \\
\hline & & \multirow[t]{3}{*}{$\begin{array}{l}\text { Whole } \\
\text { Text }\end{array}$} & $\begin{array}{l}\text { First } \\
\text { phrase }\end{array}$ & 49.34 & 46.37 \\
\hline & & & $\begin{array}{l}\text { Last } \\
\text { phrase }\end{array}$ & 48.76 & 45.71 \\
\hline & & & $\begin{array}{l}\text { Average } \\
\text { vote }\end{array}$ & 44.43 & 45.64 \\
\hline & & \multirow[t]{3}{*}{ Subsent. } & $\begin{array}{l}\text { First } \\
\text { phrase }\end{array}$ & 54.24 & 51.46 \\
\hline & & & $\begin{array}{l}\text { Last } \\
\text { phrase }\end{array}$ & 53.72 & 50.86 \\
\hline & & & $\begin{array}{l}\text { Average } \\
\text { vote }\end{array}$ & 52.86 & 49.34 \\
\hline & & \multirow[t]{3}{*}{ Phrase } & $\begin{array}{l}\text { First } \\
\text { phrase }\end{array}$ & 54.65 & 50.56 \\
\hline & & & $\begin{array}{l}\text { Last } \\
\text { phrase }\end{array}$ & 54.60 & 50.50 \\
\hline & & & $\begin{array}{l}\text { Average } \\
\text { vote }\end{array}$ & 54.44 & 50.01 \\
\hline
\end{tabular}

Table 1. Semantic affect sensing as a 3-classes problem

Table 2 shows the results of semantic affect sensing in BMRC-S as a 5-classes problem. The meanings of the column titles are the same as for Table 1.

\begin{tabular}{|c|c|c|c|c|}
\hline$B \ln$. & Gran. & Strategy & $R$ & $P$ \\
\hline \multirow{12}{*}{30.81} & \multirow[t]{3}{*}{ Majority } & $\begin{array}{l}\text { First } \\
\text { phrase } \\
\end{array}$ & 32.14 & 33.61 \\
\hline & & $\begin{array}{l}\text { Last } \\
\text { phrase }\end{array}$ & 31.49 & 32.96 \\
\hline & & $\begin{array}{l}\text { Average } \\
\text { vote }\end{array}$ & 30.75 & 32.12 \\
\hline & \multirow[t]{3}{*}{ Whole text } & $\begin{array}{l}\text { First } \\
\text { phrase } \\
\end{array}$ & 28.77 & 27.37 \\
\hline & & $\begin{array}{l}\text { Last } \\
\text { phrase }\end{array}$ & 29.08 & 28.45 \\
\hline & & $\begin{array}{l}\text { Average } \\
\text { vote }\end{array}$ & 25.49 & 26.50 \\
\hline & \multirow[t]{3}{*}{ Subsent. } & $\begin{array}{l}\text { First } \\
\text { phrase }\end{array}$ & 31.98 & 33.13 \\
\hline & & $\begin{array}{l}\text { Last } \\
\text { phrase }\end{array}$ & 31.54 & 32.49 \\
\hline & & $\begin{array}{l}\text { Average } \\
\text { vote }\end{array}$ & 31.17 & 31.93 \\
\hline & \multirow[t]{3}{*}{ Phrase } & $\begin{array}{l}\text { First } \\
\text { phrase }\end{array}$ & 32.62 & 33.40 \\
\hline & & $\begin{array}{l}\text { Last } \\
\text { phrase }\end{array}$ & 32.79 & 33.52 \\
\hline & & $\begin{array}{l}\text { Average } \\
\text { vote }\end{array}$ & 32.78 & 34.42 \\
\hline
\end{tabular}

Table 2. Semantic affect sensing as a 5-classes problem

\section{Discussion \& Future Work}

Starting from linguistic approaches and our own studies, we presented a semantic approach to affect sensing. Table 1 shows that the proposed semantic approach yields higher classification rates compared to the statistical approach. For instance, in FWF the Majority, Last phrase classification rate $47.64 \%$ is much higher than the statistical baseline recall value, $37.20 \%$. Similarly, the 3-classes affect sensing value of $54.65 \%$ in BMRC-S (Phrase, First phrase classification rate) is higher than the statistical baseline recall value, $52.34 \%$. The 5-classes affect sensing value of $32.79 \%$ in BMRC$\mathrm{S}$ (Phrase, Last phrase classification rate) is higher than the statistical baseline recall value, $30.81 \%$.

Although it is difficult to give general recommendations on the choice of a granularity and a word-spotting strategy, some suggestions can be still made: combination Majority, First phrase yields a high 3-classes classification rate for all grammatical rules in FWF $(47.20 \%)$ and a high classification rate in BMRC$\mathrm{S}(54.18 \%)$. This combination also calculates a high 5classes result in a 5-classes affect sensing (32.14\%).

Hence, future work can consider the following issues. Words can acquire an emotional meaning through the context of their use. However, the proposed system would not recognize the affective meaning of utterances correctly if this meaning depends on the emotional context of an utterance. For instance, the text It was a good book! expresses positive affect since it contains the word good. Nevertheless, if the book referred to is lost the whole text can be understood as negative. Moreover, lexical affect sensing can consider contextual 
peculiarities that can be acquired from commonsense facts [12].

This paper discussed an approach to semantic affect sensing that analyzed affect of 3 classes or 5 classes. However, the proposed approach can be generalized for affect sensing using other number of classes by adjusting the corresponding rules for differentiated linking. For instance, a semantic approach to affect sensing can define phrase patterns that interpret the semantics of four emotional classes instead of five classes as in this study.

To analyze the emotional content of a sentence a deeper analysis of conjunctions could be necessary. Let us consider an example consisting of a superordinate and a subordinate clause: Alexander is very sad even if everybody else is happy. In this case, the expression "even if" intensifies the emotional content of the superordinate clause. Thus, it does not suffice to derive the emotional meaning of the complete sentence from the emotional meaning of the single clauses only. Also dropping sub clauses would not provide an ideal solution here.

Evidently, semantic approach relies on dictionaries with emotion words. However, existing dictionaries, e.g. GI or WordNet-Affect, are not exhaustive and do contain every emotion word or phrase that has an emotional meaning. For instance, the example That's a fat lot of good! is considered to be negative since it contains an idiom a fat lot of good that has a negative meaning. However, the semantic engine in the proposed system would only detect the positive word good because it is present in the system's dictionary and oversee the negative idiom a fat lot of good since it is not stored in the system's dictionary. To overcome this shortcoming, the system should not only account for particular emotion words, but also for emotional phrases (emotional idioms). Dictionaries of emotional phrases/idioms can be found in [2] and [3].

\section{Acknowledgments}

This work was partially financed by the European Union in the CALLAS Integrate Project (http://www.callas-newmedia.eu/).

\section{References}

[1] Berardinelli, J. Berardinelli Movie Review Corpus. URL: www.reelview.net. Last accessed: 2009.

[2] Cambridge Phrasal Verbs Dictionary. Second Edition. Cambridge University Press. 2006.

[3] Cambridge Idioms Dictionary. Second Edition. Cambridge University Press. 2006.

[4] Choi, Y., Cardie, C. Learning with Compositional Semantics as Structural Inference for Subsentential Sentiment Analysis. ACL, 2008, 793-801
[5] Craggs, R. Annotating emotion in dialogue - issues and approaches. 7th Annual CLUK Research Colloquium. 2004.

[6] Engel, R. SPIN: A Semantic Parser for Spoken Dialog Systems. In Proceedings of the Fifth Slovenian And First International Language Technology Conference (IS-LTC 2006), 2006.

[7] Internet Movie Database. 2008. URL: www.imdb.com. Last accessed: 6.06.2008.

[8] Izard, C.E. Human Emotions. Plenum Press, New York, New York. 1977.

[9] Klein, D., Manning, C. D. 2003. Accurate Unlexicalized Parsing. 41st Meeting of the Association for Computational Linguistics: 423-430.

[10] Leech, G. N., Svartvik, J. A communicative grammar of English. Third edition. Longman. 2003.

[11] Levin, Beth. English verb classes and alternations. The University of Chicago Press. 1993.

[12] Liu, H., Lieberman, H., Selker, T. A Model of Textual Affect Sensing Using Real-World Knowledge. IUI-03, the 8th international conference on intelligent user interfaces: 125-132. Miami, US: ACM Press. 2003.

[13] Neviarouskaya, A., Prendinger, Ishizuka, M. Textual Affect Sensing for Social and Expressive Online Communication, Affective Computing and Intelligent Interaction (A. Pavia, P. Prada and R. W. Picard (Eds.)), (Proc. Int'l Conf. ACII2007, Lisbon, Portugal), Springer LNCS 4738: 218-229. 2007.

[14] Osherenko, A. André, E. Lexical Affect Sensing: Are Affect Dictionaries Necessary to Analyze Affect? In Proceedings of Affective Computing and Intelligent Interaction (ACII), Springer. 2007.

[15] Quirk, R., Greenbaum, S. A University Grammar of English. Longman Publishing Group; Abridged Edition. 1988.

[16] Read, J. Recognising affect in text using pointwisemutual information. Masters thesis, University of Sussex. 2004.

[17] Singh, P. 2002. The public acquisition of commonsense knowledge. Proceedings of AAAI Spring Symposium. Palo Alto, CA, AAAI. pp. 1223-1237.

[18] Stone, P. J., Dunphy, D. C., Smith, M.S., Ogilvie, D. M. The General Inquirer: A Computer Approach to Content Analysis. MIT Press. 1966.

[19] Valitutti, A., Strapparava, C., Stock, O. Developing Affective Lexical Resources. PsychNology Journal. 2/1: 61-83. 2004

[20] Wiebe, J., Riloff, E. 2005. Creating Subjective and Objective Sentence Classifiers from Unannotated Texts. Proceeding of CICLing-05, International Conference on Intelligent Text Processing and Computational Linguistics. Lecture Notes in Computer Science, vol. 3406. Mexico City, MX: Springer. pp. 475-486. 